\title{
Perspective
}

\section{Emerging role of junctophilin-2 as a regulator of calcium handling in the heart}

\author{
Alejandro GARBINO ${ }^{1,2}$, Xander HT WEHRENS ${ }^{1,2,3, *}$ \\ ${ }^{1}$ Department of Molecular Physiology and Biophysics, ${ }^{2}$ Translational Biology and Molecular Medicine Program, ${ }^{3}$ Department of \\ Medicine-Division of Cardiology, Baylor College of Medicine, Houston, Texas, USA
}

Junctophilin-2 (JPH2) is a membrane-binding protein that plays a key role in the organization of the junctional membrane complex (JMC) in cardiac myocytes. JPH2 is believed to keep the plasma membrane and sarcoplasmic reticulum at a fixed distance within the $\mathrm{JMC}$, which is essential for proper $\mathrm{Ca}^{2+}$-induced $\mathrm{Ca}^{2+}$ release during the excitation-contraction process. Recent studies have revealed that mutations in the JPH2 gene are associated with hypertrophic cardiomyopathy, highlighting the importance of this protein for normal cardiac physiology. In this paper, we review current knowledge about the structure and function of junctophilin-2 in the heart.

Keywords: $\mathrm{Ca}^{2+}{ }^{-}$induced $\mathrm{Ca}^{2+}$ release; excitation contraction coupling; heart failure; junctional membrane complex; junctophilin-2; RyR2

Acta Pharmacologica Sinica (2010) 31: 1019-1021; doi: 10.1038/aps.2010.116; published online 9 Aug 2010

Junctophilin-2 (JPH2) is a membrane-binding protein that plays an important role in junctional membrane complexes (JMC) in cardiac myocytes. There are four members in the junctophilin protein family (JPH1-4), and they are believed to bridge the physical gap between the plasma membrane (PM) and the sarcoplasmic/endoplasmic reticulum (SR/ER) in excitable cells. The JPH2 gene encodes the principal junctophilin isoform in the heart, although JPH1 is also expressed to a lesser extent ${ }^{[1]}$. JPH1 is the major isoform in skeletal muscle, whereas $\mathrm{JPH} 3$ and $\mathrm{JPH} 4$ are neuronal isoforms expressed in subsurface cisternae ${ }^{[2]}$.

A recent phylogenetic analysis of over $60 \mathrm{JPH}$ genes from over 40 species revealed that junctophilins are highly conserved, in particular the 'membrane occupation and recognition nexus' (MORN) motifs found in the N-terminus of all isoforms ${ }^{[3]}$ (Figure 1). In the case of JPH2, eight MORN domains are thought to mediate attachment to the PM, either by binding membrane lipids ${ }^{[4]}$ or proteins within the plasma membrane ${ }^{[5]}$. The 14 -amino acid MORN motifs are highly conserved across isoforms and species, suggesting that these domains are essential for JPH2 function ${ }^{[3]}$. Computational models predict the formation of a well-conserved a-helical domain of about $\sim 100$ amino acids, which is believed to provide the structural basis for the distance-spanning feature of

\footnotetext{
* To whom correspondence should be addressed. E-mail wehrens@bcm.edu

Received 2010-06-04 Accepted 2010-07-08
}

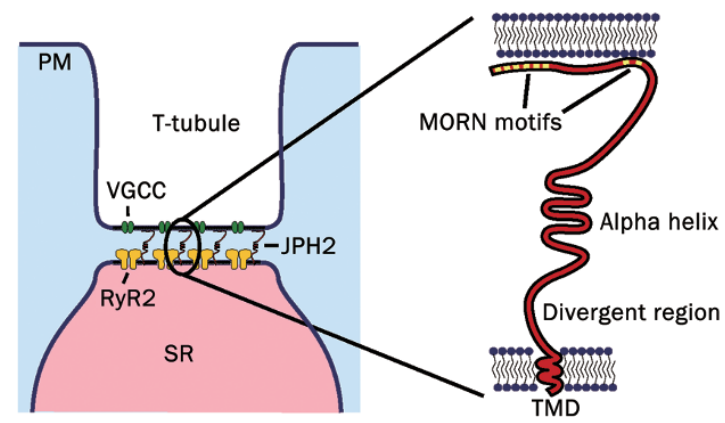

Figure 1. Schematic representation of a junctional membrane complex (JMC) in cardiac myocytes, showing voltage-gated $\mathrm{Ca}^{2+}$ channels (VGCC, also known as L-type $\mathrm{Ca}^{2+}$ channels), cardiac ryanodine receptors (RyR2), and junctophilin-2 (JPH2) proteins. The inset depicts the proposed structural domains of JPH2. PM, plasma membrane; SR, sarcoplasmic reticulum; TMD, transmembrane domain.

the protein ${ }^{[1,3]}$. Each $J P H$ isoform also contains a divergent region, that exhibits a high degree of conservation $(83 \%-91 \%)$ across species, but is poorly conserved across the $4 \mathrm{JPH}$ isoforms $(15 \%-17 \%)^{[3]}$. The function of this domain is presently unknown, although it may play a role in isoform-specific JPH functions. Finally, junctophilins are believed to bind to the ER/SR membrane using a C-terminal, 22-amino acid transmembrane anchor. At present, there is no x-ray crystallography or well-defined structural model available for JPHs.

Recent studies have begun to uncover the physiological role 
of JPH2 in cardiac muscle. Germ-line knockout of JPH2 turned out to be lethal in mice ${ }^{[1]}$. Although JPH2-/- embryos appeared to develop normally, hearts did not exhibit rhythmic contractility and embryos died by day E10.5. Electron microscopy analysis of ventricular myocytes isolated from E9.5 JPH2-/embryos revealed a severely decreased number of JMCs ${ }^{[1]}$. Moreover, these myocytes exhibited $\mathrm{Ca}^{2+}$ transients with a lower amplitude compared to wild-type controls. Finally, a large number of the JPH2- - myocytes showed $\mathrm{Ca}^{2+}$ transients that were not evoked by PM depolarization and occurred randomly, suggesting that JPH2 is required for normal SR $\mathrm{Ca}^{2+}$ release $^{[1]}$.

$\mathrm{Ca}^{2+}$-induced $\mathrm{Ca}^{2+}$ release from the $\mathrm{SR}$ is an essential component of excitation-contraction (EC) coupling and cardiac myocyte function (Figure 2) ${ }^{[6]}$. Depolarization of the PM triggers the opening of voltage-gated $\mathrm{Ca}^{2+}$ channels (VGCC), allowing influx of $\mathrm{Ca}^{2+}$ ions into the cytosol. This triggers the release of a greater amount of $\mathrm{Ca}^{2+}$ from the SR via ryanodine receptors (RyR2). After the $\mathrm{Ca}^{2+}$-induced contraction of the sarcomere, myocyte relaxation occurs when $\mathrm{Ca}^{2+}$ ions are pumped back into the SR by sarco/endoplasmic reticulum $\mathrm{Ca}^{2+}$-ATPase (SERCA2a) or $\mathrm{Ca}^{2+}$ is extruded from the myocyte by $\mathrm{Na}^{+} / \mathrm{Ca}^{2+}$ exchanger (NCX). Junctophilin is believed to be essential for normal $\mathrm{Ca}^{2+}$-induced $\mathrm{Ca}^{2+}$ release by keeping VGCC and RyR2 $\mathrm{Ca}^{2+}$ channels at a fixed distance within the $\mathrm{Ca}^{2+}$ release unit to ensure stable excitation-contraction coupling ${ }^{[1]}$.

JPH2 may also modulate $\mathrm{Ca}^{2+}$ handling by direct interac-

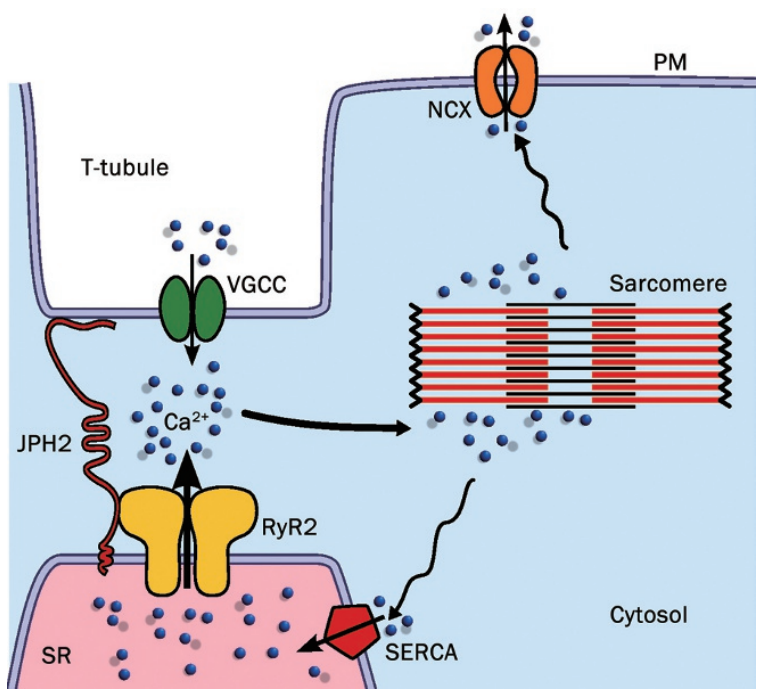

Figure 2. Overview of the flow of calcium within the junctional membrane complex during excitation-contraction coupling. Plasma membrane (PM) depolarization triggers the opening of voltage-gated $\mathrm{Ca}^{2+}$ channels (VGCC), allowing influx of calcium ions. This triggers the release of a greater amount of $\mathrm{Ca}^{2+}$ from the sarcoplasmic reticulum (SR) via ryanodine receptors (RyR2). After $\mathrm{Ca}^{2+}$-induced contraction of the sarcomere, myocyte relaxation occurs when calcium ions are pumped back into the $\mathrm{SR}$ by sarco/endoplasmic reticulum $\mathrm{Ca}^{2+}$ ATPase (SERCA) or removed from the myocyte by the $\mathrm{Na}^{+} / \mathrm{Ca}^{2+}$ exchanger (NCX). Proper structure and function of junctophilin-2 (JPH2) is believed to be essential for proper excitation-contraction coupling in cardiac myocytes. tions with $\mathrm{Ca}^{2+}$ channels. In skeletal muscle, JPH1 was shown to bind directly to the skeletal muscle isoform $\mathrm{RyR}{ }^{[7]}$. It is therefore likely that JPH2 will also bind to RyR2 in cardiac myocytes, although this remains to be confirmed experimentally. In addition, it was shown that JPH2 binds to the canonical-type transient receptor potential cation channel type 3 (TRPC3), although the physiological role of this interaction is still unknown ${ }^{[8]}$. Therefore, changes in the expression level or function of JPH2 may impact intracellular $\mathrm{Ca}^{2+}$ handling in various ways. As such, JPH2 may represent an interesting molecular target to normalize disease-induced changes in $\mathrm{Ca}^{2+}$ homeostasis ${ }^{[9]}$.

Compromised EC coupling has been postulated as a key cellular mechanism for defective cardiac contractility in failing hearts ${ }^{[10]}$. Gomez et al ${ }^{[10]}$ elegantly demonstrated that the ability of VGCC to trigger $\mathrm{Ca}^{2+}$ release from the SR via RyR2 (ie, the gain of EC coupling) was reduced in rats with heart failure. Because expression levels of VGCC and RyR2 were normal in these failing hearts, the defect was localized to the coupling between both types of $\mathrm{Ca}^{2+}$ channels. More recently, $\mathrm{Xu}$ et $a l^{[11]}$ proposed that decreased JPH2 expression might underlie defective EC coupling in rats with cardiac hypertrophy. Decreased JPH2 expression has also been reported in two mouse models of heart failure, the muscle-LIM protein knockout model of dilated cardiomyopathy and the activated H-ras transgenic mouse model of hypertrophic cardiomyopathy ${ }^{[5]}$. These results suggest that loss of JPH2 in failing hearts may contribute to defects in EC coupling, although it remains unclear whether JPH2 alterations play a primary or secondary role in the development of heart disease.

Landstrom et al ${ }^{[12]}$ recently reported mutations in the JPH2 gene in patients with hypertrophic cardiomyopathy (HCM). Three missense mutations (S101R, Y141H, and S165F) were found in 388 unrelated patients with HCM, but were absent in 500 control individuals. None of the JPH2 mutation carriers had mutations in any other known HCM-linked gene. Matsushita et al ${ }^{[13]}$ also reported a mutation in JPH2 (G505S) in a Japanese cohort of HCM patients. Expression of mutant but not wild-type JPH2 in H9c2 cells caused cellular hypertrophy ${ }^{[12]}$. Moreover, overexpression of mutant JPH2 in HL-1 cardiomyocytes attenuated the amplitude of $\mathrm{Ca}^{2+}$ transients, suggesting that the EC coupling process was disrupted in cells expressing mutant $\mathrm{JPH}^{[122]}$. Additional studies will be needed to further characterize the effects of mutant JPH2 in the context of adult cardiac myocytes. Nevertheless, these translational studies suggest that abnormal JPH2 function may lead to hypertrophy and heart failure in patients.

\section{Conclusion}

Junctophilin-2 has emerged as a potentially important regulator of excitation-contraction coupling in cardiac myocytes. Although the physiological role of JPH2 needs to be studied more extensively, it is currently believed that JPH2 plays a critical role in properly spacing and aligning VGCCs in the plasma membrane and ryanodine receptors on the sarcoplasmic reticulum. Reduced levels of JPH2 may contribute to 
defective excitation-contraction coupling in cardiac disease states such as hypertrophic cardiomyopathy and heart failure. Therefore, targeting JPH2 and its binding partners may represent a new therapeutic strategy for the treatment of heart disease.

\section{Acknowledgements}

Xander HT WEHRENS is a WM Keck Foundation Distinguished Young Scholar in Medical Research, and is supported by NIH/NHLBI grants R01-HL089598 and R01-HL091947, Muscular Dystrophy Association grant \#69238, and the Fondation Leducq Award to the "Alliance for Calmodulin Kinase Signaling in Heart Disease".

\section{References}

1 Takeshima H, Komazaki S, Nishi M, lino M, Kangawa K. Junctophilins: a novel family of junctional membrane complex proteins. Mol Cell 2000; 6: 11-22.

2 Nishi M, Sakagami H, Komazaki S, Kondo H, Takeshima H. Coexpression of junctophilin type 3 and type 4 in brain. Brain Res Mol Brain Res 2003; 118: 102-10.

3 Garbino A, van Oort RJ, Dixit SS, Landstrom AP, Ackerman MJ, Wehrens $\mathrm{XH}$. Molecular evolution of the junctophilin gene family. Physiol Genomics 2009; 37: 175-86.

4 Im YJ, Davis AJ, Perera IY, Johannes E, Allen NS, Boss WF. The $\mathrm{N}$-terminal membrane occupation and recognition nexus domain of Arabidopsis phosphatidylinositol phosphate kinase 1 regulates enzyme activity. J Biol Chem 2007; 282: 5443-52.
5 Minamisawa S, Oshikawa J, Takeshima H, Hoshijima M, Wang Y, Chien $\mathrm{KR}$, et al. Junctophilin type 2 is associated with caveolin-3 and is down-regulated in the hypertrophic and dilated cardiomyopathies. Biochem Biophys Res Commun 2004; 325: 852-6.

6 Wehrens XH, Lehnart SE, Marks AR. Intracellular calcium release and cardiac disease. Annu Rev Physiol 2005; 67: 69-98.

7 Phimister AJ, Lango J, Lee EH, Ernst-Russell MA, Takeshima H, Ma J, et al. Conformation-dependent stability of junctophilin 1 (JP1) and ryanodine receptor type 1 (RyR1) channel complex is mediated by their hyper-reactive thiols. J Biol Chem 2007; 282: 8667-77.

8 Woo JS, Hwang JH, Ko JK, Kim do H, Ma J, Lee EH. Glutamate at position 227 of junctophilin-2 is involved in binding to TRPC3. Mol Cell Biochem 2009; 328: 25-32.

9 Santonastasi M, Wehrens XH. Ryanodine receptors as pharmacological targets for heart disease. Acta Pharmacol Sin 2007; 28: 937-44.

10 Gomez AM, Valdivia HH, Cheng H, Lederer MR, Santana LF, Cannell $\mathrm{MB}$, et al. Defective excitation-contraction coupling in experimental cardiac hypertrophy and heart failure. Science 1997; 276: 800-6.

11 Xu M, Zhou P, Xu SM, Liu Y, Feng X, Bai SH, et al. Intermolecular failure of L-type $\mathrm{Ca}^{2+}$ channel and ryanodine receptor signaling in hypertrophy. PLoS Biol 2007; 5: e21.

12 Landstrom AP, Weisleder N, Batalden KB, Bos JM, Tester DJ, Ommen $\mathrm{SR}$, et al. Mutations in JPH2-encoded junctophilin-2 associated with hypertrophic cardiomyopathy in humans. J Mol Cell Cardiol 2007; 42: 1026-35.

13 Matsushita Y, Furukawa T, Kasanuki H, Nishibatake M, Kurihara $\mathrm{Y}$, Ikeda $\mathrm{A}$, et al. Mutation of junctophilin type 2 associated with hypertrophic cardiomyopathy. J Hum Genet 2007; 52: 543-8. 